Владикавказский математический журнал 2014, Том 16, Выпуск 2, С. 62-68

УДК $\mathbf{5 1 7 . 9 6 8}$

\title{
ОГРАНИЧЕННОСТЬ ПОТЕНЦИАЛА РИССА В ВЕСОВЫХ ОБОБЩЕННЫХ ГРАНД-ПРОСТРАНСТВАХ ЛЕБЕГА
}

\section{С. М. Умархаджиев}

\begin{abstract}
Доказана теорема о двухвесовой ограниченности линейных операторов во введенных нами ранее обобщенных гранд-пространствах Лебега. С помощью этой теоремы получены двухвесовые оценки нормы потенциала Рисса в рассматриваемых пространствах.
\end{abstract}

Ключевые слова: обобщенное гранд-пространство Лебега, потенциал Рисса, интерполяционная теорема, весовые оценки.

\section{1. Введение}

Неравенство Соболева [2]

$$
\left\|I^{\alpha} f\right\|_{L^{q}\left(\mathbb{R}^{n}\right)} \leqslant c\|f\|_{L^{p}\left(\mathbb{R}^{n}\right)}, \quad 1<p<q<\infty, 1 / q=1 / p-\alpha / n,
$$

для потенциала Рисса

$$
I^{\alpha} f:=\int_{\mathbb{R}^{n}}|x-y|^{\alpha-n} f(y) d y, \quad 0<\alpha<n,
$$

в пространствах Лебега $L^{p}\left(\mathbb{R}^{n}\right)$ имеет известные весовые обобщения, полученные в работах В. Кокилашвили $[1,15,16]$, Е. T. Sawyer [25], Е. T. Sawyer и R. L. Weeden [26], C. Pérez [23, 24], S. Tord [30].

В этой работе мы рассматриваем потенциалы Рисса в весовых обобщенных грандпространствах Лебега $L_{a}^{p)}\left(\mathbb{R}^{n}, w\right)$. В 1992 г. Т. Iwaniec и С. Sbordone [13] ввели grand Lebesgue spaces (гранд-пространства Лебега) по ограниченному множеству $\Omega \subset \mathbb{R}^{n}$ следующим определением:

$$
L^{p), \theta}(\Omega)=\left\{f: \sup _{0<\varepsilon<p-1} \varepsilon^{\frac{\theta}{p-\varepsilon}}\|f\|_{L^{p-\varepsilon}(\Omega)}<\infty\right\}, \quad 1<p<\infty, \theta>0 .
$$

Операторы гармонического анализа интенсивно исследовались в таких пространствах в последние годы; они продолжают привлекать внимание исследователей в связи с различными приложениями [5, 6], [8-12], [14], [17-21].

В $[27,28]$ был предложен подход, позволяющий ввести гранд-пространства Лебега на множествах неограниченной меры. В наиболее общей форме этот подход реализован в [3] в виде

$$
L_{a}^{p), \theta}(\Omega, w)=\left\{f: \sup _{0<\varepsilon<p-1} \varepsilon^{\frac{\theta}{p-\varepsilon}}\|f\|_{L^{p-\varepsilon}\left(\Omega, w a^{\varepsilon}\right)}<\infty\right\}
$$

(c) 2014 Умархаджиев С. М. 
где $1<p<\infty, \theta>0$ и $\Omega \subseteq \mathbb{R}^{n}-$ произвольное открытое множество; в случае $\theta=1$ пишут $L_{a}^{p}(\Omega, w):=L_{a}^{p, 1}(\Omega, w)$, и $L^{p, \theta}\left(\mathbb{R}^{n}, w\right)=\left.L^{p), \theta}\left(\mathbb{R}^{n}, w\right)\right|_{a \equiv 1}$ - в случае $a \equiv 1$.

Весовое обобщенное гранд-пространство Лебега $L_{a}^{p), \theta}(\Omega, w)$ зависит от «функционального параметра» $a$ и является расширением классического пространства Лебега $L^{p}(\Omega, w)$ при условии $a \in L^{p}(\Omega, w)$.

В работах $[3,27]$ с помощью теремы Рисса - Торина - Стейна - Вейса об интерполировании с изменением меры показано, что ограниченность произвольного линейного оператора в двух «близких друг к другу» обычных весовых пространствах Лебега влечет ограниченность в весовом гранд-пространстве Лебега. На основе этого в [3] доказана ограниченность операторов Кальдерона - Зигмунда и максимального оператора Харди - Литтлвуда в весовых гранд-пространствах Лебега.

Основная цель данной работы - исследование действия потенциала Рисса во введенных в [3] весовых обобщенных гранд-пространствах Лебега. Для этого мы сначала в теореме 3.1 , применением той же теремы Рисса - Торина - Стейна - Вейса получаем двухвесовые оценки для линейных операторов в рассматриваемых весовых грандпространствах. Как следствие из этих оценок и свойств весов Макенхаупта - Уидена получаем основное утверждение - теорему 4.2 об ограниченности потенциала Рисса в весовых обобщенных гранд-пространствах Лебега.

Обозначения. $C, c$ - различные абсолютные положительные постоянные, которые могут иметь различные значения даже в одной и той же строке; $\Omega$ - открытое множество в $\mathbb{R}^{n} ;|A|$ - мера Лебега измеримого множества $A \subset \mathbb{R}^{n} ; v, w-$ веса на $\Omega$, т. е. неотрицательные локально интегрируемые на $\Omega$ функции, обращающиеся в нуль на множестве нулевой меры; $w(E)=\int_{E} w(x) d x ; B(x, r)=\left\{y \in \mathbb{R}^{n}:|y-x|<r\right\} ; \widetilde{B}(x, r)=B(x, r) \cap \Omega$; $Q-$ куб в $\mathbb{R}^{n}$ с ребрами, параллельными координатным осям; $\hookrightarrow$ означает непрерывное вложение; $p^{\prime}=\frac{p}{p-1}$.

\section{2. Предварительные сведения}

2.1. Определения и вспомогательные утверждения. Через $A_{p}, 1<p<\infty$, обозначаем класс весовых функций, удовлетворяющих условию Макенхаупта [22]:

$$
\sup _{Q \subset \mathbb{R}^{n}}\left(\frac{1}{|Q|} \int_{Q} w(x) d x\right)\left(\frac{1}{|Q|} \int_{Q} w(x)^{-\frac{1}{p-1}} d x\right)^{p-1}<\infty .
$$

ОПредЕлЕНИЕ 2.1. Будем говорить, что пара весов $(w, v)$ принадлежит классу $A_{p, q}^{\alpha}$, $0<\alpha<n, 1<p, q<\infty[26]$, если

$$
\sup _{Q \subset \mathbb{R}^{n}}|Q|^{\alpha / n+1 / q-1 / p}\left(\frac{1}{|Q|} \int_{Q} v(x) d x\right)^{1 / q}\left(\frac{1}{|Q|} \int_{Q} w(x)^{1-p^{\prime}} d x\right)^{1 / p^{\prime}}<\infty .
$$

ОПРЕДЕЛЕНИЕ 2.2. Будем говорить, что пара весов $(w, v)$ принадлежит классу $A^{*}(p, q), 1<p, q<\infty[7]$, если

$$
\sup _{Q \subset \mathbb{R}^{n}}\left(\frac{1}{|Q|} \int_{Q} v(x)^{q} d x\right)^{1 / q}\left(\frac{1}{|Q|} \int_{Q} w(x)^{-p^{\prime}} d x\right)^{1 / p^{\prime}}<\infty .
$$


Лемма 2.3. Пусть $0<\alpha<n, 1<p, q<\infty$ и выполнены условия

1) для весовых функций $w$ и $v$ существуют числа $r>1$ и $0<\varepsilon<q-1$ такие, что

$$
\sup _{Q}|Q|^{\alpha / n+1 /(q-\varepsilon)-1 / p}\left(\int_{Q} v(x)^{r} d x\right)^{\frac{1}{(q-\varepsilon) r}}\left(\int_{Q} w(x)^{\left(1-p^{\prime}\right) r} d x\right)^{\frac{1}{p^{\prime} r}}<\infty
$$

2) неотрицательные функции $a$ и $b$ таковы, что существует число $\delta>\varepsilon$ такое, что $\left(a^{\frac{\delta}{p}}, b^{\frac{\delta}{q-\varepsilon}}\right) \in A^{*}(p, q-\varepsilon)$.

Тогда $\left(w a^{\varepsilon}, v b^{\varepsilon}\right) \in A_{p, q-\varepsilon}^{\alpha}$.

$\triangleleft$ Обозначим

$$
\Delta:=\sup _{Q \subset \mathbb{R}^{n}}|Q|^{\alpha / n+1 /(q-\varepsilon)-1 / p}\left(\frac{1}{|Q|} \int_{Q} v(x) b(x)^{\varepsilon} d x\right)^{1 /(q-\varepsilon)}\left(\frac{1}{|Q|} \int_{Q}\left[w(x) a(x)^{\varepsilon}\right]^{1-p^{\prime}} d x\right)^{1 / p^{\prime}} .
$$

K обоим интегралам применим неравенство Гёльдера с показателем $r>1$. Получим

$$
\begin{aligned}
\Delta \leqslant & \sup _{Q \subset \mathbb{R}^{n}}|Q|^{\alpha / n+1 /(q-\varepsilon)-1 / p}\left(\frac{1}{|Q|} \int_{Q} v(x)^{r} d x\right)^{\frac{1}{r(q-\varepsilon)}}\left(\frac{1}{|Q|} \int_{Q} w(x)^{\left(1-p^{\prime}\right) r} d x\right)^{\frac{1}{r p^{\prime}}} \\
& \times\left[\left(\frac{1}{|Q|} \int_{Q}\left[b(x)^{\varepsilon r^{\prime} /(q-\varepsilon)}\right]^{q-\varepsilon} d x\right)^{\frac{1}{q-\varepsilon}}\left(\frac{1}{|Q|} \int_{Q}\left[a(x)^{\varepsilon r^{\prime} / p}\right]^{-p^{\prime}} d x\right)^{\frac{1}{p^{\prime}}}\right]^{1 / r^{\prime}} .
\end{aligned}
$$

Откуда следует утверждение леммы. $\triangleright$

В случае $0<\alpha<n, 1<p<n / \alpha, 1 / q=1 / p-\alpha / n$ известно соотношение

$$
\left(w^{p / q}, w\right) \in A_{p, q}^{\alpha} \Longleftrightarrow w \in A_{1+q / p^{\prime}} .
$$

\section{2. Ограниченность риссова потенциала в весовых пространствах Лебе-}

га. Будем говорить, что вес $w$ удовлетворяет условию удвоения, если существует $C>0$ такое, что $w(2 Q) \leqslant C w(Q)$ для всех кубов $Q \subset \mathbb{R}^{n}$ с ребрами, параллельными координатным осям.

Основное утверждение данной статьи получено посредством теоремы Сойера - Уидена [26] об ограниченности оператора потенциала $I^{\alpha}$ в классических пространствах Лебега:

Теорема 2.4. Пусть $0<\alpha<n, 1<p \leqslant q<\infty$.

(1) Неравенство

$$
\left\|I^{\alpha} f\right\|_{L^{q}\left(\mathbb{R}^{n}, v\right)} \leqslant c\|f\|_{L^{p}\left(\mathbb{R}^{n}, w\right)}
$$

выполняется, если существует $r>1$ такое, что

$$
\sup _{Q}|Q|^{\alpha / n+1 / q-1 / p}\left(\int_{Q} v(x)^{r} d x\right)^{1 /(q r)}\left(\int_{Q} w(x)^{\left(1-p^{\prime}\right) r} d x\right)^{1 /\left(p^{\prime} r\right)}<\infty .
$$

(2) Если предположить, что $p<q$ и функции $v$ и $w^{1-p^{\prime}}$ удовлетворяют условию удвоения, то (4) выполняется тогда и только тогда, когда $(w, v) \in A_{p, q}^{\alpha}$. 
2.3. Интерполяционная теорема. Нам понадобится следующая интерполяционная теорема Рисса - Торина - Стейна - Вейса с изменением меры $[4,29]$, которую мы формулируем в весовых терминах.

Теорема 2.5. Пусть $p_{k}, q_{k} \in[1, \infty)$ и $v_{k}, w_{k}$ - веса на $\Omega, k=1,2$, и $T-$ сублинейный оператор, определенный на $L^{p_{1}}\left(\Omega, w_{1}\right) \cap L^{p_{2}}\left(\Omega, w_{2}\right)$. Если $T: L^{p_{1}}\left(\Omega, w_{1}\right) \hookrightarrow L^{q_{1}}\left(\Omega, v_{1}\right)$ с нормой $M_{1}$ и $T: L^{p_{2}}\left(\Omega, w_{2}\right) \hookrightarrow L^{q_{2}}\left(\Omega, v_{2}\right)$ с нормой $M_{2}$, то

$$
T: L^{p_{t}}\left(\Omega, w_{t}\right) \hookrightarrow L^{q_{t}}\left(\Omega, v_{t}\right)
$$

с нормой $M \leqslant M_{1}^{1-t} M_{2}^{t}$, где

$$
\begin{aligned}
& \frac{1}{p_{t}}=\frac{1}{p_{1}}+\left(\frac{1}{p_{2}}-\frac{1}{p_{1}}\right) t, \quad \frac{1}{q_{t}}=\frac{1}{q_{1}}+\left(\frac{1}{q_{2}}-\frac{1}{q_{1}}\right) t, \\
& w_{t}=w_{1}^{(1-t) \frac{p_{t}}{p_{1}}} w_{2}^{t \frac{p_{t}}{p_{2}}}, \quad v_{t}=v_{1}^{(1-t) \frac{q_{t}}{q_{1}}} v_{2}^{t \frac{q_{t}}{q_{2}}}, \quad 0<t<1 .
\end{aligned}
$$

\section{3. Ограниченность линейных операторов в обобщенных гранд-пространствах Лебега}

Теорема 3.1. Пусть $1<p, q<\infty, w$ и $v$ - два веса на $\Omega \subseteq \mathbb{R}^{n}$. Пусть линейный оператор $T$ ограничен из пространства $L^{p}(\Omega, w)$ в пространство $L^{q}(\Omega, v)$ и ограничен из пространства $L^{p_{0}}\left(\Omega, w a^{p-p_{0}}\right)$ и пространство $L^{q_{0}}\left(\Omega, v b^{q-q_{0}}\right)$ для некоторых двух чисел $1<p_{0}<p, 1<q_{0}<q$ и некоторых двух неотрицательных на $\Omega$ функций $a \in L^{p}(\Omega, w)$ и $b \in L^{q}(\Omega, v)$.

Тогда оператор $T$ ограничен из весового обобщенного гранд-пространства Лебега $L_{a}^{p), \theta}(\Omega, w)$ в весовое обобщенное гранд-пространство Лебега $L_{b}^{q), \theta q / p}(\Omega, v)$.

$\triangleleft$ Для произвольного положительного числа $\theta_{1}$ имеем

$$
\|T f\|_{L_{b}^{q), \theta_{1}}(\Omega, v)}=\sup _{0<\varepsilon<q-1} \varepsilon^{\frac{\theta_{1}}{q-\varepsilon}}\|T f\|_{L^{q-\varepsilon}\left(\Omega, v b^{\varepsilon}\right)}=\max \{A, B\},
$$

где

$$
A=\sup _{0<\varepsilon \leqslant q-q_{0}} \varepsilon^{\frac{\theta_{1}}{q-\varepsilon}}\|T f\|_{L^{q-\varepsilon}\left(\Omega, v b^{\varepsilon}\right)}, \quad B=\sup _{q-q_{0}<\varepsilon<q-1} \varepsilon^{\frac{\theta_{1}}{q-\varepsilon}}\|T f\|_{L^{q-\varepsilon}\left(\Omega, v b^{\varepsilon}\right)} .
$$

Оценка величины $A$. По интерполяционной теореме 2.5 с параметрами

$$
p_{1}=p, \quad p_{2}=p_{0}, \quad q_{1}=q, \quad q_{2}=q_{0}, \quad v_{1}=w, \quad v_{2}=w a^{p-p_{0}}, \quad w_{1}=v, \quad w_{2}=v b^{q-q_{0}}
$$

получаем, что

$$
\|T f\|_{L^{q-\varepsilon}\left(\Omega, v b^{\varepsilon}\right)} \leqslant M_{1}^{1-t} M_{2}^{t}\|f\|_{L^{p_{\varepsilon}\left(\Omega, w a^{p-p_{\varepsilon}}\right)}},
$$

$p_{\varepsilon}=\left(\frac{1}{p}+t\left(\frac{1}{p_{0}}-\frac{1}{p}\right)\right)^{-1}, t=\frac{\varepsilon q_{0}}{\left(q-q_{0}\right)(q-\varepsilon)}$ для всех $\varepsilon \in\left[0, q-q_{0}\right]$. Следовательно,

$$
A \leqslant \sup _{0<\varepsilon \leqslant q-q_{0}} M_{1}^{1-t} M_{2}^{t} \varepsilon^{\frac{\theta_{1}}{q-\varepsilon}}\|f\|_{L^{p_{\varepsilon}}\left(\Omega, w a^{p-p_{\varepsilon}}\right)}
$$

$$
\leqslant \sup _{0<\varepsilon \leqslant q-q_{0}} M_{1}^{1-t} M_{2}^{t} \varepsilon^{\frac{\theta_{1}}{q-\varepsilon}}\left(p-p_{\varepsilon}\right)^{-\frac{\theta}{p_{\varepsilon}}} \sup _{0<p-p_{\varepsilon} \leqslant p-p_{0}}\left(p-p_{\varepsilon}\right)^{\frac{\theta}{p_{\varepsilon}}}\|f\|_{L^{p_{\varepsilon}}\left(\Omega, w a^{p-p_{\varepsilon}}\right)} .
$$


Учитывая, что $t=\frac{p_{0}\left(p-p_{\varepsilon}\right)}{p_{\varepsilon}\left(p-p_{0}\right)}$, получим $A \leqslant C\left(p_{0}, q_{0}\right)\|f\|_{L_{a}^{p), \theta}(\Omega, w)}$, где

$$
C\left(p_{0}, q_{0}\right)=\sup _{0<t \leqslant 1} M_{1}^{1-t} M_{2}^{t}\left(\frac{t q\left(q-q_{0}\right)}{q_{0}+t\left(q-q_{0}\right)}\right)^{-\theta\left(\frac{1-t}{q}+\frac{t}{q_{0}}\right)}\left(\frac{t p\left(p-p_{0}\right)}{p_{0}+t\left(p-p_{0}\right)}\right)^{\theta_{1}\left(\frac{1-t}{p}+\frac{t}{p_{0}}\right)} .
$$

Выделив из правой части сомножитель $t^{\theta_{1} \frac{1}{q}-\theta \frac{1}{p}}$, можно сделать вывод: константа $C\left(p_{0}, q_{0}\right)$ конечна, если $\theta_{1}=\frac{q}{p} \theta$.

Оценка величины $B$. Воспользовавшись неравенством Гёльдера с показателем $\frac{q_{0}}{q-\varepsilon}>1$, получим

$$
\|T f\|_{L^{q-\varepsilon}\left(\Omega, v b^{\varepsilon}\right)} \leqslant\|b\|_{L^{q}(\Omega, v)}^{q\left(\frac{1}{q-\varepsilon}-\frac{1}{q_{0}}\right)}\|T f\|_{L^{q_{0}\left(\Omega, v b^{q-q_{0}}\right)}}
$$

так что

$$
\begin{gathered}
B \leqslant \sup _{q-q_{0}<\varepsilon<q-1} \varepsilon^{\frac{\theta_{1}}{q-\varepsilon}}\|b\|_{L^{q}(\Omega, v)}^{q\left(\frac{1}{q-\varepsilon}-\frac{1}{q_{0}}\right)}\|T f\|_{L^{q_{0}}\left(\Omega, v b^{q-q_{0}}\right)} \\
=\left(q-q_{0}\right)^{-\frac{\theta_{1}}{q_{0}}}\|b\|_{L^{q}(\Omega, v)}^{-\frac{q}{q_{0}}} \sup _{q-q_{0}<\varepsilon<q-1} \varepsilon^{\frac{\theta_{1}}{q-\varepsilon}}\|b\|_{L^{q}(\Omega, v)}^{\frac{q}{q-\varepsilon}}\left(q-q_{0}\right)^{\frac{\theta_{1}}{q_{0}}}\|T f\|_{L^{q_{0}}\left(\Omega, v b^{q-q_{0}}\right)} \\
\leqslant \inf _{0<q-q_{0}<q-1}\left(h^{-1}\left(q-q_{0}\right) \sup _{q-q_{0}<\varepsilon<q-1} h(\varepsilon)\right) \cdot A=A .
\end{gathered}
$$

где обозначено $h(\varepsilon):=\varepsilon^{\frac{\theta_{1}}{q-\varepsilon}}\|b\|_{L^{q}(\Omega, v)}^{\frac{q}{q-\varepsilon}}$. Объединяя оценки для $A$ и $B$, получим утверждение теоремы 3.1. $\triangleright$

ЗАмечАниЕ 3.2 . В случае $\frac{p_{0}}{q_{0}}=\frac{p}{q}$ справедливо $C\left(p_{0}, q_{0}\right)=\max \left\{M_{1}\left(\frac{q}{p}\right)^{\frac{\theta}{p}}, M_{2}\left(\frac{q}{p}\right)^{\frac{\theta}{p_{0}}}\right\}$.

\section{4. Основное утверждение}

В работе [21] получено необходимое и достаточное условие ограниченности потенциала Рисса в весовом гранд-пространстве Лебега на отрезке $[0,1]$ :

Теорема 4.1. Пусть $0<\alpha<1,1<p<\infty, \theta>0$ и $q=p /(1-\alpha p)$. Тогда неравенство

$$
\left\|I^{\alpha}\left(f w^{\alpha}\right)\right\|_{L^{q), \theta(1+\alpha q)}([0,1], w)} \leqslant c\|f\|_{L^{p), \theta}([0,1], w)}
$$

выполняется тогда и только тогда, когда $w \in A_{1+q / p^{\prime}}$.

На основании теоремы 3.1 мы можем получить двухвесовую оценку нормы потенциала Рисса по $\mathbb{R}^{n}$ в весовых обобщенных гранд-пространствах Лебега:

Теорема 4.2. Пусть $0<\alpha<n$ и $1<p<q<\infty$. Пусть

1) $(w, v) \in A_{p, q}^{\alpha}$;

2) $v$ и $w^{1-p^{\prime}}$ удовлетворяют условию удвоения;

3) для некоторых чисел $r>1$ и $0<\varepsilon_{0}<q-1$ выполняется условие

$$
\sup _{Q}|Q|^{\alpha / n+1 /\left(q-\varepsilon_{0}\right)-1 / p_{0}}\left(\int_{Q} v(x)^{r} d x\right)^{\frac{1}{\left(q-\varepsilon_{0}\right) r}}\left(\int_{Q} w(x)^{\left(1-p_{0}^{\prime}\right) r} d x\right)^{\frac{1}{p_{0}^{\prime} r}}<\infty, \quad p_{0} \leqslant q-\varepsilon_{0} ;
$$

4) неотрицательные функции $a$ и $b$ таковы, что существует число $\delta>\varepsilon_{0}$ такое, что $\left(a^{\frac{\delta}{p_{0}}}, b^{\frac{\delta}{q-\varepsilon_{0}}}\right) \in A^{*}\left(p_{0}, q-\varepsilon_{0}\right)$ и функции $v b^{\varepsilon_{0}}$ и $\left(w a^{\varepsilon_{0}}\right)^{1-p_{0}^{\prime}}$ удовлетворяют условию удвоения. 
Тогда оператор Рисса $I^{\alpha}$ ограничен из обобщенного гранд-пространства Лебега $L_{a}^{p, \theta}\left(\mathbb{R}^{n}, w\right)$ в обобщенное гранд-пространство Лебега $L_{b}^{q, \theta q / p}\left(\mathbb{R}^{n}, v\right), \theta>0$.

$\triangleleft$ Из условий 1) и 2) в силу теоремы 2.4 следует ограниченность оператора Рисса из пространства $L^{p}(\Omega, w)$ в пространство $L^{q}(\Omega, v)$, из условий 3$)$ и 4$)$ на основании леммы 2.3 и теоремы 2.4 следует его ограниченность из пространства $L^{p_{0}}\left(\Omega, w a^{\varepsilon_{0}}\right)$ в пространство $L^{q-\varepsilon_{0}}\left(\Omega, v b^{\varepsilon_{0}}\right)$. Таким образом, условия теоремы 3.1 выполнены и тем самым теорема 4.2 доказана. $\triangleright$

Следствие 4.3. Пусть $\Omega \subseteq \mathbb{R}^{n}, 0<\alpha<n, 1<p<n / \alpha, 1 / q=1 / p-\alpha / n, w \in A_{1+q / p^{\prime}}$ и функции $w$ и $w^{-p^{\prime} / q}$ удовлетворяют условию удвоения.

Тогда $I^{\alpha}: L^{p, \theta}\left(\Omega, w^{p / q}\right) \hookrightarrow L^{q, \theta q / p}(\Omega, w)$.

$\triangleleft$ Из соотношения $(3)$ следует, что пара функций $\left(w^{p / q}, w\right)$ удовлетворяет условию 1$)$ теоремы 4.2. Следовательно, $w \in A_{1+\frac{q}{p^{\prime}}}$ Существует число $\varepsilon>0$ такое, что $w \in A_{1+\frac{q}{p^{\prime}}-\varepsilon}$. Решив систему уравнений $\frac{q-\varepsilon_{0}}{p_{0}^{\prime}}=\frac{q}{p^{\prime}}-\varepsilon, \frac{1}{q-\varepsilon_{0}}=\frac{1}{p_{0}}-\frac{\alpha}{n}$, получим числа $p_{0}$ и $\varepsilon_{0}$, для которых имеет место соотношение $A_{1+\frac{q}{p^{\prime}}}=A_{1+\frac{q-\varepsilon_{0}}{p_{0}^{\prime}}}$. Откуда следует выполнимость условия 3 ) теоремы 4.2. Первая часть условия 4) выполняется, так как класс $A_{p, q}^{*}$ содержит константу, а вторая - совпадает с условием 2). Таким образом, все условия теоремы 4.2 выполнены и следствие доказано. $\triangleright$

Отметим, что в случае отрезка $[0,1]$ теорема 4.2 содержит достаточную частью теоремы 4.1 при выборе $w=w^{1-\alpha p}, v=w, b=1, a=w^{\alpha}$.

Автор выражает искреннюю благодарность профессору С. Г. Самко за полезные замечания, способствовшие улучшению содержания статьи.

\section{Литература}

1. Кокилашвили В. Максимальные функции и интегралы типа потенциала в весовых пространствах Лебега и Лоренца // Тр. Мат. ин-та АН CCCP.-1985.-Vol. 172.-C. 192-201.

2. Соболев C. Л. Об одной теореме функционального анализа // Мат. сб.-1938.-Vol. 4 (3).-C. 471497.

3. Умархаджиев С. М. Обобщение понятия гранд-пространства Лебега // Изв. вузов. Математика.2014.-Vol. 4.-C. 42-51.

4. Bergh J., Löfström J. Interpolation Spaces. An Introduction.-Berlin: Springer, 1976.-207 p.

5. Capone C., Fiorenza A. On small Lebesgue spaces // J. Function Spaces and Appl.-2005.-Vol. 3.P. 73-89.

6. Di Fratta G., Fiorenza A. A direct approach to the duality of grand and small Lebesgue spaces // Nonlinear Analysis: Theory, Methods and Applications.-2009.-Vol. 70, № 7.-P. 2582-2592.

7. Ding Y., Lin C.-C. Two-weight norm inequalities for the rough fractional integrals // Int. J. Math. Math. Sci.-2001.-Vol. 25, № 8.-P. 517-524.

8. Fiorenza A., Gupta B., Jain P. The maximal theorem in weighted grand Lebesgue spaces // Studia Math.-2008.-Vol. 188, № 2.-P. 123-133.

9. Fiorenza A. Duality and reflexivity in grand Lebesgue spaces // Collect. Math.-2000.-Vol. 51, № 2.P. 131-148.

10. Fiorenza A., Karadzhov G. E. Grand and small Lebesgue spaces and their analogs // J. Anal. Appl.2004.-Vol. 23, № 4.-P. 657-681.

11. Fiorenza A., Rakotoson J. M. Petits espaces de Lebesgue et leurs applications // C.R.A.S. t.-2001.Vol. 333.-P. 1-4.

12. Greco L., Iwaniec T., Sbordone C. Inverting the $p$-harmonic operator // Manuscripta Math.-1997.Vol. 92.-P. 249-258.

13. Iwaniec T., Sbordone C. On the integrability of the Jacobian under minimal hypotheses // Arch. Rational Mech. Anal.-1992.-Vol. 119.-P. 129-143.

14. Kokilashvili V., Meskhi A. A note on the boundedness of the Hilbert transform in weighted grand Lebesgue spaces // Georgian Math. J.-2009.-Vol. 16, № 3.-P. 547-551. 
15. Kokilashvili $V$. Two-weighted estimates for some integral transforms in Lebesgue spaces with mixed norm and imbedding theorems // Georgian Math. J.-1994.-Vol. 1, № 5.-P. 495-503.

16. Kokilashvili $V$. On a progress in the theory of integral operators in weighted Banach function spaces // Function Spaces, Differential Operators and Nonlinear Analysis. Proc. of the Conf. held in Milovy, Bohemian-Moravian Uplands, May 28 - June 2, 2004.-Praha: Math. Inst. Acad. Sci. Czech Republick, 2005.-P. 152-175.

17. Kokilashvili $V$. Boundedness criterion for the Cauchy singular integral operator in weighted grand Lebesgue spaces and application to the Riemann problem // Proc. A. Razmadze Math. Inst.-2009.Vol. 151.-P. 129-133.

18. Kokilashvili $V$. The Riemann boundary value problem for analytic functions in the frame of grand $L^{p)}$ spaces // Bull. Georgian Nat. Acad. Sci.-2010.-Vol. 4, № 1.-P. 5-7.

19. Kokilashvili V., Samko S. Boundedness of weighted singular integral operators on a Carleson curves in Grand Lebesgue spaces // ICNAAM 2010: Intern. Conf. Numer. Anal. Appl. Math.-Vol. 1281.P. 490-493.

20. Kokilashvili V., Samko S. Boundedness of weighted singular integral operators in Grand Lebesgue spaces // Georg. Math. J.-2011.-Vol. 18, № 2.-P. 259-269.

21. Meskhi A. Criteria for the boundedness of potential operators in grand Lebesgue spaces.arXiv:1007.1185.

22. Muckenhoupt B. Weighted norm inequalities for the Hardy maximal function // Trans. Amer. Math. Soc.-1972.-Vol. 165.-P. 207-226.

23. Pérez $C$. Two weighted norm inequalities for Riesz potetials and uniform $L^{p}$-weighted Sobolev inequalities // Indiana Univ. Math. J.-1990.-Vol. 39, № 1.-P. 31-44.

24. Pérez $C$. Two weighted inequalities for potetial and fractional type maximal operators // Indiana Univ. Math. J.-1994.-Vol. 43, № 2.-P. 663-683.

25. Sawyer E. T. A two weight weak type inequality for fractional integrals // Trans. Amer. Math. Soc.1984.-Vol. 281, № 1.-P. 339-345.

26. Sawyer E. T., Weeden R. L. Weighted inequalities for fractional integrals on euclidean and homogeneous spaces // Amer. J. Math.-1992.-Vol. 114, № 4.-P. 813-874.

27. Samko S. G., Umarkhadzhiev S. M. On Iwaniec-Sbordone spaces on sets which may have infinite measure // Azerb. J. Math.-2011.-Vol. 1, № 1.-P. 67-84.

28. Samko S. G., Umarkhadzhiev S. M. On Iwaniec-Sbordone spaces on sets which may have infinite measure: addendum // Azerb. J. Math.-2011.-Vol. 1, № 2.-P. 143-144.

29. Stein E. M., Weiss G. Interpolation of operators with change of measures // Trans. Amer. Math. Soc.-1958.-Vol. 87.-P. 159-172.

30. Tord $S$. Weighted norm inequalities for Riesz potentials and fractional maximal functions in mixed norm Lebesgue spaces // Stud. Math.-1990.-Vol. 97, № 3.-P. 239-244.

Статъя поступила 29 июня 2013 г.

УМАРХАДЖИЕв САЛАУДИН МУСАЕВИч

Чеченский государственный университет, профессор каф. информационных технологий

РОССИЯ, 364037, Грозный, ул. Киевская, 33

E-mail: umsalaudin@gmail.com

\title{
BOUNDEDNESS OF THE RIESZ POTENTIAL OPERATOR IN WEIGHTED GRAND LEBESGUE SPACES
}

\author{
Umarkhadzhiev S. M.
}

We prove a theorem on the two-weighted boundedness of linear operators in generalized Grand Lebesgue spaces introduced in our previous papers. This theorem is applied to obtain two-weigt estimated for the Riesz potential operator in such spaces.

Key words: generalized grand Lebesgue space, Riesz potential operator, interpolation theorem. 